Zbl Arbeitsmed 2015 $\cdot 65: 160$

DOI 10.1007/s40664-015-0017-0

Online publiziert: 9. Mai 2015

(c) Springer-Verlag Berlin Heidelberg 2015

\section{Busch ${ }^{1} \cdot$ S. Zitnik ${ }^{2}$ - D.A. Groneberg ${ }^{2} \cdot$ M. Schwarzer ${ }^{2}$}

${ }^{1}$ Institut für Arbeitsmedizin, Charité - Universitätsmedizin Berlin, Berlin, Deutschland

${ }^{2}$ Institut für Arbeitsmedizin, Sozialmedizin und Umweltmedizin, Universitätsklinikum, Goethe-Universität Frankfurt am Main, Frankfurt, Deutschland
In diesem Beitrag wird im Abschnitt „Bezug zur Arbeitsmedizin“ zur Frage einer erforderlichen Pflichtvorsorge als Rechtsbezug die Biostoffverordnung in der Fassung vom 18.12.2008 genannt, in der eine Pflichtvorsorge für Tetanus nicht aufgeführt sei. Seit 2008 ist die Rechtsgrundlage für die arbeitsmedizinische Vorsorge, auch bei Biostoffen, aber bekanntlich die ArbMedVV. Die BioStoffV vom 18.12.2008 enthält daher auch im $\$ 15$ nur noch den Hinweis auf die seitdem für die arbeitsmedizinische Vorsorge geltende ArbMedVV.

Die Angabe im Literaturverzeichnis hinsichtlich der aktuellen Fassung der BioStoffV ist ebenfalls veraltet. Korrekt wäre der 15.07.2013.

Es fehlt auch der Hinweis, dass mit Neufassung der ArbMedVV vom
23.10.2013 nun unter bestimmen Rahmenbedingungen auch ein Impfangebot bei Angebots- oder Wunschvorsorge auszusprechen ist.

Wir bitten, die korrekten Angaben zu beachten und die Fehler zu entschuldigen.

Die Redaktion

\section{Korrespondenzadresse}

Prof. Dr. D.A. Groneberg

Institut für Arbeitsmedizin, Sozialmedizin und Umweltmedizin

Universitätsklinikum, Goethe-Universität Frankfurt am Main

Theodor-Stern-Kai 7, 60590 Frankfurt

occup-med@uni-frankfurt.de 